\title{
Study of He II Raman Scattering Profiles in NGC 7027
}

\author{
D. Péquignot \\ Observatoire de Paris - Meudon, F-92195 Meudon Principal Cedex, \\ France
}

J. R. Walsh

Space Telescope European Co-ordinating Facility, European Southern Observatory, Karl-Schwarzschild Strasse 2 , D-85748 Garching, Germany

C. Morisset

Institut d'Astrophysique de Marseille, Traverse du Siphon, BP 8, F-13376 Marseille Cedex 12, France

Péquignot et al. (1997) reported the discovery of a broad spectral feature to the blueward of $\mathrm{H} \beta$, centred at a wavelength of $4852 \AA$, in NGC 7027 . This was conclusively shown to arise from the Raman scattering of $\mathrm{He}^{++}(2-8) \mathrm{UV}$ photons by neutral $\mathrm{H}$, and was the first detection in a planetary nebula. Raman scattering features had previously been observed in symbiotic stars: Raman scattering of O VI by neutral H was discovered by Schmid (1992) and also found for He II by van Groningen (1993). The large width of the resulting feature is mainly due to the conservation of the line width in frequency during the scattering process.

The occurrence of a high ionization nebula as a source for $\mathrm{He}^{++}$photons proximate to a neutral hydrogen shell at the interface with the surrounding molecular cloud creates the condition for Raman scattering of He II. The redshift of the spectral feature relative to the expected value (4851 1 ) was attributed to the expansion of the neutral $\mathrm{H}$ shell, with respect to the He II zone of NGC 7027. In PN, Raman lines provide new diagnostics for: direct indication of presence of $\mathrm{H} 0$; kinematic information on $\mathrm{H} 0$ relative to He II; determination of the Raman conversion efficiency. Neutral (atomic) $21 \mathrm{~cm} \mathrm{H}$ emission can be determined from radio data, but in practice the observations for Galactic PN are difficult on account of the high optical depth of Galactic H emission. Taylor et al. (1990) detected absorption by neutral $\mathrm{H}$ in five $\mathrm{PN}$ with one showing emission as well.

High signal-to-noise and high spectral resolution observations have now been conducted of the Raman multiplet He II $4851 \AA$ in NGC 7027 at a grid of seven slit positions in order to map the spatial extent of the Raman lines. The WHT and ISIS spectrometer with a holographic 2400 lines/mm grating was employed. The strength of the Raman feature at $4851 \AA$ increases, relative to $\mathrm{H} \beta$ and He II $4686 \AA$, towards the outer regions of the nebula, consistent with scattering of He II photons by the surrounding neutral shell. Integrating the strength of the $4851 \AA$ feature, a map of the Raman line strength formed from 
the multi-slit observations is presented which shows directly for the first time the extent of $\mathrm{H}^{0}$ in this prototype nebula. The diameter of the neutral shell is measured as about $9 \times 11^{\prime \prime}(\mathrm{E}-\mathrm{W}$ by $\mathrm{N}-\mathrm{S})$. Comparison with the $\mathrm{H}_{2}$ map of Latter et al. (2000) shows that the neutral $\mathrm{H}$ shell peaks somewhat inside the sharp $\mathrm{H}_{2}$ shell.

A relatively strong He II Raman line was also detected at $6546 \AA$ in red spectra of NGC 7027, but is badly blended with the strong [N II] $6548 \AA$ line. Deblending of this feature from the [N II] line serves to confirm the Raman identification of $\lambda 4852 \AA$. Detection of a weak He II Raman feature is also indicated for the first time in the vicinity of $\mathrm{H} \gamma$, at a wavelength $4332 \AA$, bringing to three the number of He II Raman features found in NGC 7027.

A dedicated search for the existence of He II Raman $4851 \AA$ in a number of control objects, where the line would not be expected on the basis of the model, has also been performed. No detection of He II Raman lines were made in low ionization nebulae (e.g. NGC 6572) and high ionization nebulae without evidence for a neutral shell (e.g. NGC 7662). A spectrometric search of several high ionization nebulae with evidence for neutral shells (e.g. emission of neutral ions) was also undertaken. The $4851 \AA$ Raman feature was detected in NGC 6886 and NGC 6302, showing it to be a common occurrence in high ionization nebulae with evidence for neutral material, dust and molecular emission.

\section{References}

Latter, W. B., Dayal, A., Bieging, J. H., Meakin, C., Hora, J. L., Kelly, D. M., Tielens, A. G. G. M., 2000, ApJ, 529, 783

Péquignot, D., Baluteau, J.-P., Morisset, C., Boisson, C., 1997, A\&A, 217, 222

Schmid, H. M., 1992, A\&A, 254, 224

Taylor, A. R., Gussie, G. T., Pottasch, S. R., 1990, ApJ, 351, 515

van Groningen, E., 1993, MNRAS, 264, 975 